\title{
Balance de la asociación entre América Latina y el Caribe y la Unión Europea. La nueva agenda*
}

Assessment of the alliance between Latin America and the European Union. The new agenda

\section{Lourdes García Rodríguez ${ }^{* *}$ Fernando Jiménez Valderrama***}

\begin{abstract}
RESUMEN
La Primera Cumbre $L A C-U E$, realizada en Río de Janeiro en 1999, fue el comienzo de una alianza estratégica entre América Latina y el Caribe y la Unión Europea. A pesar de las dificultades, esta asociación ha sido útil para ambas partes. En este artículo estudiamos la evolución de estas relaciones birregionales analizando su futuro inmediato. También presentamos las siete cumbres birregionales entre 1999 y 2013, los progresos obtenidos de ellas, los desafios que la alianza estratégica debe enfrentar, el debate del futuro de las relaciones birregionales y las nuevas estrategias que la asociación ALC-UE debe seguir para alcanzar sus objetivos. Finalmente, proponemos una nueva agenda y temas para consolidar la cooperación regional.
\end{abstract}

PALABRAS CLAVE: Alianza Estratégica América Latina y el Caribe-Unión Europea, relaciones birregionales ALC-UE, acuerdo de asociación, tratados de libre comercio, cooperación, integración económica.

\begin{abstract}
The FirSt LAC-EU Summit, carried out in Rio de Janeiro in 1999, was the beginning of a strategic alliance between Latin America and the European Union. Despite the difficulties, this association has been useful for both parties. In this article, we study the evolution of these biregional relationships analyzing its immediate future. We also present the 7 bi-regional summits from 1999 until 2013, the progress made by them, the challenges that this strategic alliance has to face, the debate of the future of bi-regional relationships and the new strategies that the LAC-EU association must follow to achieve its objectives. Finally, we propose a new agenda and issues to consolidate the regional cooperation.
\end{abstract}

KEY WORDS: Strategic Alliance between European Union and Latin America, LAC-EU bi-regional relationships, association agreement, free trade agreement, cooperation, economic integration.

\footnotetext{
* Recibido: 11 de febrero de 2013. Aceptado: 25 de marzo de 2013.

** Profesora de Geopolítica en la facultades de Relaciones Internacionales y de Ciencia Política y Gobierno de la Universidad del Rosario, Colombia (garciarod.maria@urosario.edu.co).

*** Jefe del Área de Derecho Privado y de la Empresa en la Facultad de Derecho y Ciencias Politicas de la Universidad de La Sabana, Colombia (fernando.jimenez@unisabana.edu.co).
} 


\section{Sumario}

1. Introducción

2. La Alianza Estratégica de ALC y la UE

A) Las cumbres birregionales

B) Balance de la de Asociación Estratégica entre ALC-UE

3. Retos que enfrenta la Alianza Estratégica entre ALC-UE

A) Un contexto internacional cambiante

B) La nueva dinámica económica y comercial de América Latina y el Caribe

c) Las dinámicas internas de integración europea y latinoamericana

4. Nuevos temas en la agenda de la asociación de la CELAC y la UE

5. Conclusiones

\section{Introducción}

A pesar de los altibajos que las relaciones entre América Latina y el Caribe (ALC) y la Unión Europea (UE) han mantenido en las dos últimas décadas, puede decirse que, por expreso deseo de ambas partes, éstas continúan avanzando a diferentes velocidades dependiendo de los países, como veremos a lo largo del artículo. La dinámica creada en las relaciones entre ALC y uE ha permitido abrir las vías de una Alianza Estratégica entre dos regiones que, a pesar de ser muy dispares en lo socioeconómico, tienen vínculos históricos y culturales muy fuertes que, de hecho, han hecho posible llegar a posiciones y acciones conjuntas en asuntos de interés para ambas partes, tanto a nivel birregional como multilateral.

A lo largo de este artículo trataremos de mostrar los logros y las sombras del marco de asociación creado entre ambas regiones, a partir de la Alianza Estratégica establecida en la 1 Cumbre Birregional ALC-UE celebrada en Río de Janeiro (Brasil) en 1999. En primer lugar nos centraremos en analizar la evolución y balance de esta Alianza hasta el momento actual, a través de sus diferentes etapas, destacando el papel de cada una de las cumbres celebradas, para después hacer 8 un balance de los avances y acuerdos conseguidos en los últimos catorce años. Nuestro objetivo es mostrar que, a pesar de las dificultades, la asociación ha sido fructífera y útil para ambas partes.

En segundo lugar pretendemos mostrar los retos y desafíos a los que se enfrenta la Alianza Estratégica entre ALc y la UE en la coyuntura actual, protagonizada, por un lado, por la crisis financiera iniciada en Estados Unidos y los importantes efectos negativos en la economía y finanzas de la UE, y por otro lado, por el reposicionamiento de países y regiones a nivel mundial (caso de Asia y ALc, regiones especialmente atractivas por su crecimiento económico, el 
dinamismo de las inversiones y el aumento de sus flujos comerciales). Ello nos permitirá abordar el debate acerca del futuro de las relaciones birregionales y las estrategias o reequilibrios de fuerzas que deben permitir avanzar en la asociación de ALC y la Ue.

En tercer y último lugar analizaremos, desde una perspectiva pragmática, la nueva agenda y los temas que la deben protagonizar. Se trata de ampliar y consolidar la cooperación entre ambas regiones en áreas que marcarán el futuro no sólo de las relaciones birregionales, sino de las economías y las sociedades de sus respectivos países, así como su posición en el mundo. En este sentido, la complementariedad y la horizontalidad (el equilibrio entre ambas regiones) parecen ser las claves que deberían marcar el futuro de las relaciones entre ALC y la UE.

\section{La Alianza Estratégica de ALC y la UE}

La primera cumbre entre la ALc y la uE se celebró en 1999 en Río de Janeiro (Brasil) y estableció como objetivo prioritario el inicio de un proceso que permitiría alcanzar una Asociación Estratégica Birregional basada en tres pilares: el diálogo político, la cooperación y el libre comercio. ${ }^{1}$

Desde entonces, y haciendo un primer y rápido avance, podríamos decir que la relación birregional entre ALC y la UE sigue un camino lento pero útil. Las relaciones subregionales han tenido un desigual y lento caminar durante este periodo, especialmente en las negociaciones con el Mercosur y la Comunidad Andina de Naciones (CAN), y se han consolidado con la firma de diferentes acuerdos de asociación y tratados de libre comercio.

En el caso concreto de México, la relación bilateral con la UE es estrecha, fluida y altamente institucionalizada, que se inicia con la firma, en 1997, del Acuerdo de asociación económica, concertación política y cooperación (TLCUEM, primer acuerdo de este tipo firmado por la UE con un país latinoamericano) ${ }^{2} \mathrm{y}$ consolidada, desde 2008, con la firma de un Acuerdo de Asociación Estratégica ${ }^{3}$ y un Plan Ejecutivo Conjunto, México y la UE, ${ }^{4}$ que han permitido estrechar, en-

\footnotetext{
1 Véase Declaración de Río de Janeiro. Disponible en: http://eulacfoundation.org/es/documentos/declaraci\%C3\%B3nde-r\%C3\%ADo-1999.

2 Un "acuerdo global" que entró en vigor en 2000 y que abarcaba el diálogo político, los intercambios comerciales y la cooperación.

${ }^{3}$ com (2008) 447 final. Comunicación de la Comisión al Consejo y al Parlamento Europeo. Hacia una Asociación Estratégica ue-México, Bruselas, 15 de julio de 2008. Disponible en: http://eeas.europa.eu/mexico/docs/com08_447_ es.pdf.

${ }^{4}$ 9820/10 PRESSE 126, Consejo de la Unión Europea. Asociación Estratégica México-Unión Europea. Plan Ejecutivo Conjunto. Comillas, 16 de mayo de 2010. Disponible en: http://register.consilium.europa.eu/pdf/es/10/st09/st09820. es10.pdf.
} 
tre ambas partes, lazos de confianza, diálogo fluido y profundizar en diferentes ámbitos de cooperación. ${ }^{5}$

Antes de abordar el debate que pretende redefinir las actuales relaciones birregionales debemos hacer un recorrido sobre las principales aportaciones de las cumbres celebradas en la última década, con la finalidad de tener una visión de conjunto que permita hacer un balance real sobre la evolución de la Alianza Estratégica suscrita entre ambas regiones. ${ }^{6}$

\section{A) Las cumbres birregionales}

La celebración de las cumbres birregionales ha servido para estrechar las relaciones entre los países de ALC y la UE sobre la base de unos valores compartidos (la democracia y la defensa del multilateralismo en las relaciones internacionales, el Estado de derecho y la seguridad jurídica), así como para construir una asociación estratégica que privilegia la cooperación entre ambas regiones. En este sentido, la diplomacia de las cumbres se ha transformado en un elemento clave de las estrategias políticas y económicas de los países de ambas regiones, especialmente en un contexto en el que hay gran interés por encontrar socios estratégicos con los cuales avanzar en el escenario mundial y multilateral. Analicemos a continuación las diferentes etapas de la Alianza Estratégica ALC-UE y los resultados de cada una de las cumbres birregionales celebradas.

\section{a) Primera etapa (1999-2004). Cumbres de Rio, Madrid $y$ Guadalajara: grandes expectativas y pocos resultados concretos}

Los años finales de la década de los noventa del siglo pasado se caracterizaron por el auge de los procesos de integración regional, protagonizados, en el

\footnotetext{
${ }^{5}$ En diferentes foros y medios se ha publicado el interés de ambas partes por ampliar el TLCuEM a lo largo de 2013, introduciendo diferentes temas, como por ejemplo, un "capítulo agropecuario", además de asuntos relacionados con el desarrollo de una "nueva economía basada en el conocimiento" y "temas de modernidad tecnológica" como las relaciones con las tecnologías de la información y el comercio electrónico, que en su dia no fueron abordados. Disponible en: http://www.americaeconomia.com/economia-mercados/comercio/mexico-y-la-ue-evaluaran-suacuerdo-de-asociacion-economica, http://www.quadratinmexico.com/ue-por-actualizar-acuerdo-de-asociacioncon-mexico/, http://www.economia.gob.mx/eventos-noticias/informacion-relevante/9468-boletin042-13.

${ }^{6}$ Véase Arenas, Gonzalo y Casanova, Héctor (ed.). "La Asociación Estratégica alc-ue. Balance de una década y desafíos futuros", en La Unión Europea, América Latina y el Caribe. Diez años de alianza estratégica, Celare, Chile, 2010, pp. 29-50; PEÑA, FéuX. Las Cumbres alC-UE: su origen y evolución, su relevancia, su potencial. Consideraciones para una reflexión colectiva en función de la próxima Cumbre de Madrid, SEgiB, Madrid, 2010, y SberRo, Stephan. "Las tres etapas de la relación estratégica birregional entre América Latina, el Caribe y la Unión Europea", en Bases renovadas para la relación Unión Europea, América Latina y el Caribe (Actas del Seminario UE-LAC/GIGA, 17 y 18 de septiembre de 2012), UELAC, Hamburgo, 2013, pp. 38-43.
} 
ámbito latinoamericano, por un Mercosur que por entonces se mostraba como el modelo más ambicioso de integración regional en ALc, los avances andinos y centroamericanos, y, en el ámbito europeo, por la firma de los Tratados de Maastricht y Ámsterdam, así como la entrada en vigor de la Unión Monetaria y el nacimiento de una moneda común, el Euro.

Éste pareció ser el contexto idóneo para que dos regiones (que decian compartir los mismos valores de democracia y economía social del mercado, además de raíces históricas y culturales marcadas) quisieran protagonizar el primer modelo de relación birregional del mundo; proclamando, en la Cumbre de Río de 1999, un nuevo birregionalismo bajo el modelo de una Asociación Estratégica entre ALC y la UE.

En las siguientes dos Cumbres (Madrid y Guadalajara) los resultados fueron desiguales. Mientras que la Declaración de Madrid ${ }^{7}$ (2002) sólo contenía palabras de compromiso para, entre otros asuntos, aumentar los esfuerzos en cooperación birregional y promover el crecimiento económico entre ambas regiones; en la Declaración de Guadalajara ${ }^{8}$ (2004) se definieron tres temas como ejes de la Cumbre (multilateralismo, cohesión social y relación birregional), y se consiguió concretar un proyecto propiamente birregional con la adopción del programa EUROSOciAL, cuyo objetivo era promover intercambios de experiencias, conocimiento especializado y buenas prácticas en el campo social entre las dos regiones, especialmente en educación y salud, fundamentales para aumentar la cohesión social ${ }^{9}$ en ambas regiones.

b) Segunda etapa (2006-2008). La transición: las Cumbres de Viena y Lima

Esta etapa estuvo protagonizada por las dificultades y cambios que se produjeron durante estos años, tanto en el entorno de la UE como en ALc, y que modificaron el panorama birregional. Hace parte del contexto fáctico en esta etapa:

1) Los problemas surgidos en las economías europeas como consecuencia de los efectos de la crisis financiera de los Estados Unidos;

2) La consolidación de un nuevo proyecto de integración latinoamericana liderado por Cuba y Venezuela (la llamada Alianza Bolivariana para los Pueblos de Nuestra América-Tratado de Comercio de los Pueblos, AlBA-

\footnotetext{
${ }^{7}$ Disponible en: http://eulacfoundation.org/es/documentos/declaraci\%c3\%в3n-de-madrid-2002.

${ }^{8}$ Disponible en: http://eulacfoundation.org/es/documentos/declaraci\%с3\%в3n-de-guadalajara-2004.

9 Disponible en: http://www.eurosocial-ii.eu/.
} 
тсP), ${ }^{10}$ que logró la paulatina adhesión de diferentes países del entorno latinoamericano y caribeño como Ecuador, Bolivia, Nicaragua, Antigua y Barbados, entre otros;

3) La cada vez mayor dificultad para fijar, entre los propios países latinoamericanos, estrategias económicas y comerciales conjuntas, y

4) Los escasos avances en las negociaciones de la UE con la can y el Mercosur.

En este cambiante contexto se celebraron las Cumbres de Viena y de Lima, con aportaciones de interés que conviene considerar. En la Declaración de Viena"11 (2006), además de reafirmarse la intención de intensificar los esfuerzos para definir posiciones comunes y acciones concertadas entre ambas regiones, se ampliaron los ejes básicos de discusión -incluyendo temas como terrorismo, medio ambiente, drogas y delincuencia organizada, energía, crecimiento y empleo, cooperación para el desarrollo, migración, acuerdos de asociación, integración regional, comercio, conectividad, entre otros-, y se apoyaron las propuestas para crear la Asamblea Euro-Latinoamericana ${ }^{12}$ y la Secretaría General Iberoamericana (SEGlB). ${ }^{13}$

Dos años más tarde, en la Cumbre de $\operatorname{Lima}^{14}$ (2008), las deliberaciones se centraron principalmente en la promoción de acciones a nivel nacional, regional e internacional para abordar puntos claves para ambas regiones: la pobreza, la desigualdad y la necesidad de sociedades más inclusivas (tema prioritario para los países de ALc), y el desarrollo sostenible, el medio ambiente, el cambio climático y la energía (prioritario para la UE). También se consolidó el compromiso de fortalecer la infraestructura de comunicaciones y reducir la brecha digital, al amparo de programas como@lis, ${ }^{15}$ y la ampliación de la interconexión entre las redes Clara, ${ }^{16}$ Géant $^{17}$ y CKLN, ${ }^{18}$ con una importante proyección en los ámbitos académico y tecnológico.

Además, se acordó la puesta en marcha de un segundo proyecto de carácter birregional, EUrocLIMA, ${ }^{19}$ un programa medioambiental conjunto ALC-UE, enfocado especialmente en el cambio climático y con tres objetivos principales: compartir

\footnotetext{
${ }_{10}$ Disponible en: http://www.alba-tcp.org/.

"Disponible en: http://eulacfoundation.org/es/documentos/declaraci\%c3\%в3n-de-viena-2006.

${ }_{12}$ Disponible en: http://www.europarl.europa.eu/intcoop/eurolat/menu_es.htm.

${ }_{13}$ Disponible en: http://segib.org/.

${ }_{14}$ Disponible en: http://eulacfoundation.org/es/documentos/declaraci\%C3\%B3n-de-lima-2008.

${ }^{15}$ Disponible en: http://ec.europa.eu/europeaid/where/latin-america/regional-cooperation/alis/documents/final_ publication_es.pdf.

${ }^{16}$ Disponible en: http://www.redclara.net/.

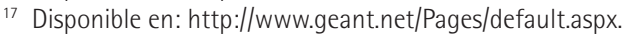

${ }^{18}$ Disponible en: http://www.ckln.org/home/.

19 Disponible en: http://www.euroclima.org/.
} 
conocimiento, fomentar un diálogo y asegurar sinergias y coordinación de acciones en este tema.

Finalmente, se decidió considerar la creación de una Fundación UE-LAC, concebida como estímulo para deliberar sobre estrategias comunes y acciones orientadas al fortalecimiento de la asociación birregional y aumentar su visibilidad, asunto que, como veremos, se concretó en la Cumbre de Madrid, dos años más tarde. En donde no hubo ningún avance fue en el ámbito de las negociaciones birregionales para la firma de los Acuerdos de Asociación de la UE con la can y el Mercosur. Sino al contrario, veremos que tras los avances en las relaciones bilaterales entre la ue y países como México, Chile y Brasil, algunos otros -como Perú y Colombia- empezaron a avanzar solos en su relación con la UE.

c) Tercera etapa: la Cumbre de Madrid (2010), entre el birregionalismo $y$ el bilateralismo

La VI Cumbre ALC-UE, celebrada en Madrid, consiguió revertir la tendencia a la baja de las relaciones birregionales y recuperar el impetu original de la Cumbre de Río, volviendo a colocar la Alianza Estratégica como punto sustancial de la relación birregional.

Madrid fue el escenario de un doble éxito, combinando proyecciones políticas de largo plazo con resultados concretos; significándose, por ello, como el inicio de una nueva etapa en las relaciones birregionales ALC-UE, ${ }^{20}$ tal como veremos a continuación.

La Declaración de Madrid, ${ }^{21}$ además de reafirmar el acervo de principios, valores e intereses comunes de la Asociación Estratégica, propuso como novedad un Plan de Acción 2010-2012, con medidas concretas, financiadas y definidas mediante una agenda práctica en ámbitos prioritarios como el desarrollo del espacio UE-ALC del conocimiento, el fomento de la interconectividad y la intensificación de los diálogos temáticos, así como la cooperación sobre migraciones, drogas, educación y cohesión social. ${ }^{22}$ Este Plan debía ser evaluado en la Cumbre de Santiago a celebrarse dos años después.

\footnotetext{
20 Véase, entre otros, SAnahuja, José Antonio. "Las relaciones entre la ue y América Latina y el Caribe tras la Cumbre de Madrid: el fin de un ciclo político y la necesidad de una nueva estrategia", en Anuario lberoamericano, Agencia efe y Fundación Real Instituto El Cano, Madrid, 2011, pp. 23-44, y Arenal, Celestino y Sanahuja, José Antonio. La cumbre alC-Ue de Madrid: un nuevo impulso a las relaciones birregionales, Fundación Carolina-AECID, Madrid, 2010. Disponible en: http://www.fundacioncarolina.es/es-ES/nombrespropios/Documents/NpArenalySanahuja1005.pdf.

${ }_{21}$ Disponible en: $\mathrm{http}: / /$ eulacfoundation.org/es/documentos/declaracion-de-madrid-0.

22 Disponible en: http://eulacfoundation.org/es/documentos/plan-de-acci\%C3\%B3n-de-madrid-2010.
} 
También se alcanzaron acuerdos para la creación - por la UE- de un Nuevo Mecanismo de Inversión en América Latina (LAIF), con el objeto de servir de palanca para movilizar fondos de respaldo a las inversiones en América Latina -tanto públicos como privados-, que ayuden a avanzar en ámbitos prioritarios como las infraestructuras y redes de comunicación, y la Fundación América Latina y el Caribe-UE (EU-LAC) con sede en Hamburgo, anunciada ya en la Cumbre de Viena. ${ }^{23}$

La Cumbre de Madrid confirmó la flexibilidad alcanzada en el contexto eurolatinoamericano para la negociación de otro tipo de acuerdos no birregionales. Se trataba de un reconocimiento de la intensificación de las relaciones bilaterales entre los diferentes países de ambas regiones frente a la, hasta ahora, protagónica relación birregional. Reconocimiento y satisfacción (expresada explícitamente en la Declaración) por los "avances conseguidos en los diferentes escenarios abiertos”: un Acuerdo Multipartes entre la UE-Colombia y Perú, un Acuerdo de Asociación entre la UE y Centroamérica, la consolidación de las Asociaciones estratégicas de la UE con México y Brasil, así como el establecimiento de la Asociación para el Desarrollo y la Innovación entre la uE y Chile.

\section{d) Cuarta etapa: la Cumbre de Santiago (2013): ¿hacia una redefinición de las relaciones?}

A finales de enero de 2013, los países de ALC y de la UE se reunieron en Santiago (Chile) en una nueva Cumbre Birregional en el marco de la recién creada Comunidad de Estados Latinoamericanos y Caribeños (CELAC). ${ }^{24}$ El tema central fue renovar y profundizar la Asociación Estratégica en torno al tema “Alianza

\footnotetext{
${ }^{23}$ Disponible en: http://eulacfoundation.org/es.

24 Las Declaraciones de Cancún en febrero de 2010 (disponible en: http://www.parlatino.org/images/stories/inicio/ celac/declaracion-cancun.pdf) y de Caracas en diciembre de 2011 (disponible en: http://www.parlatino.org/images/ stories/inicio/celac/declaracion-caracas.pdf) establecieron la creación de la cELAc como un organismo intergubernamental de ámbito regional, constituido por los jefes de Estado y de Gobierno de los paises de América Latina y el Caribe, que se propone convertir en un foro de toma de decisiones independientes en la región latinoamericana y caribeña, y en el mecanismo representativo de la región, al objeto de promover sus intereses y objetivos de integración y desarrollo. En palabras de Michael Shifter, presidente de Diálogo Interamericano, "la constitución de la celac es la última expresión del regionalismo, que tiene una larga historia en América Latina y en el Caribe. Pero una mirada más cercana sobre la CELAC, la cual no incluye a EEUu ni a Canadá, también revela que hay profundas diferencias entre los miembros sobre asuntos fundamentales relacionados tanto con la gobernabilidad como sobre los enfoques de las políticas económicas y sociales. La CELAC puede cumplir con un objetivo político, pero carece de una infraestructura institucional [...] Es muy pronto todavía para ver si realmente llega a tener una mayor institucionalización [...] Es tentadora la tesis de la bifurcación en América Latina. Para varios analistas la clave de lo que está pasando hoy en la región está en la separación entre el Pacífico y el Atlántico, que incluyen al Mercosur y la ALBA. Esta formulación puede ser un tanto simplista, pero claramente hay un bloque que tiene economías considerablemente más abiertas que el otro. Para lograr la meta de una integración regional de América Latina sería preferible que se apunte a un
} 
para el Desarrollo Sustentable: Promoción de Inversiones de Calidad Social y Ambiental". 25

Entre diciembre de 2012 y enero de 2013 la ciudad de Santiago fue sede de una serie de reuniones de alto nivel entre diferentes representantes de las dos regiones. El complejo entramado de reuniones preparatorias, anteriores a la celebración de la Cumbre, refleja la fluidez del diálogo y la coordinación a la que se ha logrado llegar entre las dos regiones: reuniones de altos funcionarios, encargados de organizar y negociar los preparativos y acuerdos a considerar en la Cumbre; reuniones sectoriales por temas en los cuales las dos regiones llevan tiempo colaborando de manera fluida y coordinada (ciencia y tecnología, migraciones, mecanismo de coordinación y cooperación en materia de drogas y reunión ministerial de economía), y eventos complementarios a la Cumbre, de carácter tan variado y representativo de las sociedades civiles de ambas regiones como el vi Encuentro Sindical UE-ALC, el vil Encuentro de la Sociedad Civil Organizada UE-AL, la I Cumbre Judicial CELAC-UE, la I Cumbre Académica de la CELAC-UE, la vı Asamblea EURoLAt, la ıv Cumbre Empresarial cELAC-UE y el vi Foro Eurolatinoamericano de Sociedad Civil. 26

La Cumbre y las declaraciones que allí se escucharon por parte de los presidentes latinoamericanos reflejan claramente el nuevo discurso que los países de ALC pretenden establecer en el ámbito de sus relaciones con la UE - basado en el reequilibrio de las relaciones-, instando a sentar las bases de una nueva relación birregional con "menos dependencia y mayor simetría".

En este sentido, los gobernantes latinoamericanos destacaron los avances sociales y económicos de sus países en los últimos años, y reclamaron un nuevo trato con los países europeos.

América Latina llega con diez años de crecimiento, de fortalecimiento de la democracia y del Estado de derecho prácticamente en todos los países que integran esta comunidad [...]. Queremos sentar las bases para una relación de mayor colaboración y mayor simetría [...], más libertad, más innovación, emprendimiento y libertad de comercio y menos proteccionismo [...].

Fueron algunas de las ideas expresadas por el anfitrión de la Cumbre, el presidente chileno Sebastián Piñera, en la clausura de la iv Cumbre Empresarial entre

rumbo único y más coherente, pero esta no es la realidad hoy". Disponible en: http://www.eldeber.com.bo/septimodia/2013-04-28/ver.php?id=130427102609.

${ }_{25}$ Disponible en: http://www.minrel.gob.cl/prontus_minrel/site/artic/20130131/asocfile/20130131162552/declaracion_de_santiago_final_26_enero.pdf.

26 Disponible en: http://www.minrel.gob.cl/prontus_minrel/site/artic/20110708/pags/20110708111726.php. 
la cELAC y la UE, que precedió a la de los jefes de Estado y de Gobierno de las dos regiones.

En ese mismo foro empresarial, el presidente colombiano, Juan Manuel Santos, reafimó esta posición, “[...] ambas regiones se necesitan mutuamente. En América Latina tenemos energía, capacidad para aumentar la producción de alimentos, y recursos de todo tipo [...], pero necesitamos tecnología y otras cosas de Europa”.

Por su parte, el presidente mexicano, Enrique Peña Nieto, aseguró que su país:

[...] seguirá siendo una economía abierta, promotora del comercio mundial y garante de las inversiones nacionales y extranjeras. El Gobierno que encabezo quiere ser un facilitador de las inversiones $\mathrm{y}$, ante todo, respetuoso de la propiedad privada [...], hay certidumbre política para las inversiones. México ofrece hoy no sólo estabilidad económica y certidumbre, sino también un mercado abierto y confiable para hacer negocios. ${ }^{27}$

Este deseo de replantear las relaciones quedó plasmado también en la Declaración final de la Cumbre:

Estamos seguros que este nuevo enfoque resultará en una relación aún más equilibrada, eficiente, constructiva y simétrica con complementariedad y solidaridad entre las dos regiones. Reafirmamos la importancia de confiar en un diálogo socialmente constructivo, inclusivo y diverso para lograr los compromisos descritos en esta Declaración (punto 3 de la Declaración de Santiago).

Los acuerdos y compromisos sustanciales logrados en la Cumbre de Santiago reflejan los avances en la relación birregional ALC-UE y muestran el grado de coordinación, fluidez y profundidad alcanzado. Entre ellos destacamos los siguientes:

- La adopción de políticas que promuevan el comercio y la inversión entre países de la cELAC y la UE, basadas en la cooperación y la complementariedad, en la solidaridad y la inclusión social, en la responsabilidad ambiental, en la equidad y la igualdad de oportunidades, en beneficios mutuos y teniendo en cuenta los diferentes niveles de desarrollo y las prioridades respectivas de los países de la cELAC y la UE.

- La asunción de acciones que faciliten y profundicen acuerdos comerciales, la integración de cadenas productivas, la transferencia de tecnología con

Disponible en: http://www.celacue2013.cl/documentos/. 
la participación de micro, pequeñas y medianas empresas, así como actores locales e indígenas, según las circunstancias nacionales en el comercio birregional.

- La extensión del programa Euroclima a una segunda fase, a la que la región caribeña podrá asociarse, en línea con la recientemente adoptada Estrategia uE Caribe.

- La profundización del Plan de Acción birregional a través de la incorporación de nuevos capítulos sobre género e inversión en el Plan de Acción 2013-2015 de la UE-CELAC aprobado.

- El reconocimiento de las negociaciones celebradas a nivel bilateral y subregional entre los países de la CELAC y la UE en el periodo 2010-2012, y que refleja la importancia y fluidez de estos contactos:

- La firma y los procesos de ratificación respectivos del Acuerdo de Libre Comercio de largo alcance entre la UE y Colombia y Perú;

- La firma y los procesos de ratificación respectivos del Acuerdo de Asociación entre Centro América y la uE;

- La negociación de un Acuerdo de Cooperación Cultural entre la UEColombia y Perú;

- Las negociaciones para modernizar el Acuerdo de Asociación Chile-UE, después de diez años de asociación;

- Las negociaciones para la actualización integral del Acuerdo de Asociación Económica, Concertación Política y Cooperación entre la UE y México, y la extensión del Plan Ejecutivo Conjunto de la Asociación Estratégica.

- Las negociaciones para la profundización de la Alianza Estratégica entre la ue y Brasil, establecida en 2007.

- La conclusión de las negociaciones y aprobación de la Estrategia de Asociación Conjunta Caribe-uE y el establecimiento del Mecanismo de Inversión del Caribe para apoyar las inversiones en el Caribe;

- La conclusión del proceso de ratificación del Acuerdo can-UE sobre Diálogo Político y Cooperación.

- La adopción de una hoja de ruta para la implementación de la Iniciativa Conjunta de lnvestigación e lnnovación para contribuir a un nuevo dinamismo en la construcción del Área de Conocimiento de la UE-CELAC.

- La publicación del Primer Compendio Estadístico sobre Migración de la CELAC-UE, considerada una valiosa fuente de información para formular 
políticas públicas basadas en el conocimiento cualitativo y cuantitativo de las tendencias de migración birregional.

- La creación del Mecanismo de Inversión para el Caribe y el fortalecimiento del Mecanismo de Inversión para América Latina, para mejorar la integración, infraestructuras de transporte y energía, eficiencia energética, energías renovables, redes de comunicación sustentables y para promover el desarrollo sustentable a través de los servicios sociales fortalecidos y un mayor apoyo a las PYMEs.

Finalmente, en el Plan de acción CELAC-UE 2013-2015, ${ }^{28}$ aprobado también en la Cumbre de Santiago, se incluyeron dos capítulos nuevos (junto con un programa de trabajo y de resultados esperados), cuyo cumplimiento será revisado en la próxima cumbre birregional a celebrar en Bruselas en 2015.

El primero de los capítulos novedosos es el de "Género", cuyos objetivos principales son darle prioridad al tema de género en el contexto de las relaciones birregionales y resaltar la voluntad política de ambas regiones de garantizar la igualdad de género y la protección, ejercicio y promoción de los derechos de la mujer.

El segundo es el de "Inversiones y emprendimiento para el desarrollo sustentable", cuyos objetivos son: a) promover inversiones birregionales de calidad social y medioambiental que contribuyan a generar mayores flujos comerciales, creación de trabajo, transferencia de tecnología, fomento de la innovación, ingresos fiscales, apoyo y desarrollo de industrias auxiliares; b) promover el emprendimiento como fuerza motriz del desarrollo económico y social, y c) facilitar las condiciones que propicien el emprendimiento y la innovación, remuevan obstáculos, desarrollen capacidades y aumenten la competitividad de las micro, pequeñas y medianas empresas (MPYME) y nuevos actores de la economía.

\section{B) Balance de la Asociación Estratégica entre ALC-UE}

El recorrido que hemos hecho de los catorce años de relaciones birregionales ALC-UE, institucionalizadas a través de seis cumbres, nos ha permitido observar los diferentes progresos logrados y la evolución de la Alianza Estratégica entre ambas regiones.

En este sentido, consideramos que la estrategia birregional con la UE sigue siendo útil y deseable para los países de ALc, ya que les está permitiendo proyec-

\footnotetext{
${ }^{28}$ Disponible en: http://www.consilium.europa.eu/uedocs/cms_Data/docs/pressdata/EN/foraff/135043.pdf; http:// eulacfoundation.org/es/documentos/plan-de-acci\%C3\%B3n-de-santiago-2013.
} 
tar el papel de la región y sus prioridades (a veces también sus diferencias) ante terceros, consolidar sus estrategias de negociación ante los 27 países de la UE, armonizar entre sí sus posiciones a través del diálogo y hacerlas compatibles con sus propios proyectos subregionales, sin que por ello se deje de avanzar en temas de la agenda bilateral y tomar decisiones concretas al respecto.

Respecto al balance de los objetivos definidos en la Asociación Estratégica que inició su camino en la Cumbre de Río de Janeiro, en 1999, destacamos tres campos principales: a) el diálogo político a diferentes niveles relacionado con la integración regional; $b$ ) el comercio, y c) la cohesión social. Todos ellos respaldados por la cooperación birregional y por la consecución de una serie de avances positivos que han reforzado el diálogo y la coordinación en diferentes ámbitos desarrollados entre los países de ALC y la UE - político, comercial, económico, tecnológico, académico y social-. Hagamos un recuento de aquéllos conseguidos a nivel birregional:

- El Espacio ALC-UE del conocimiento en materia de enseñanza superior.

- El programa alßan de enseñanza superior, que fomenta el intercambio académico entre ambas regiones a través de becas.

- La implementación de la Iniciativa Conjunta de lnvestigación e lnnovación para contribuir a un nuevo dinamismo en la construcción del Área de Conocimiento de la UE-CELAC.

- El programa EURosocial, que busca superar las inequidades y fomentar la cohesión social en la región latinoamericana.

- El programa euroclima para el intercambio de experiencias en materia de cambio climático y su extensión a una segunda fase, a la que la región caribeña se asociará.

- El fortalecimiento de la infraestructura de comunicaciones y redes digitales entre las dos regiones a través del Programa@lis y la ampliación de la interconexión entre las redes Clara, Géant y CKLN.

- La creación y fortalecimiento del Mecanismo de Inversión para América Latina, para mejorar la integración, infraestructuras de transporte y energía, eficiencia energética, energías renovables, redes de comunicación sustentable y para promover el desarrollo sustentable a través de los servicios sociales fortalecidos y un mayor apoyo a las PYMEs.

- La creación de la Asamblea Parlamentaria Euro-Latinoamericana (EuroLat).

- La creación de la Fundación EUROLAc, con sede en Hamburgo, para supervisar los avances de la Asociación Estratégica birregional. 
- La publicación del Primer Compendio Estadístico sobre Migración de la CELAC-UE, considerada una valiosa fuente de información para formular políticas públicas basadas en el conocimiento cualitativo y cuantitativo de las tendencias de migración birregional.

La Alianza Estratégica Birregional se vio reforzada a partir de la Cumbre de Madrid (2010) por la implementación de unos planes de acción, cuyos fines son tratar de consolidar líneas concretas de actuación y ampliar los ámbitos de trabajo conjunto entre los países de Alc y la UE. En este sentido, se destacan los siguientes:

- El Plan de Acción ALC-UE 2010-2012, con medidas concretas financiadas y definidas mediante una agenda concreta y práctica en ámbitos prioritarios como el desarrollo del espacio UE-ALC del conocimiento, el fomento de la interconectividad y la intensificación de los diálogos temáticos y la cooperación sobre migraciones, drogas, educación y cohesión social.

- El Plan de Acción celac-UE 2013-2015, incorporando dos nuevos capítulos sobre "Género e inversiones y emprendimiento para el desarrollo sustentable”.

En el caso del diálogo político entre los países de ALC y la UE, éste se ha ido institucionalizando a través de los años, incluso en algunos casos con anterioridad a la primera Cumbre ALC-UE de 1999. Los principales foros de diálogo y cooperación surgidos en el ámbito eurolatinoamericano son los siguientes:

- Reuniones Ministeriales, de Altos Funcionarios y Expertos en diferentes ámbitos (medio ambiente, educación, ciencia y tecnología, drogas, migración, entre otros).

- Asamblea Parlamentaria EuroLat.

- Foro Sindical UE-ALC.

- Foro Empresarial ALC-UE.

- Foro de Autoridades Locales UE-ALC.

- Foro de la Sociedad Civil Organizada UE-ALC.

- Foro Eurolatinoamericano de la Sociedad Civil.

- Foro Judicial CELAC-UE.

- Foro Académico de la celac-Ue.

- Fundación eUrolac. 
Otro de los aspectos más destacados del balance de la Alianza Estratégica entre ALC-UE ha sido la tendencia, consolidada en las últimas cumbres birregionales, de que éstas sirvan no sólo para concitar reuniones a nivel birregional, sino también para tratar temas relacionados con las agendas subregionales y bilaterales. El fin de todas estas reuniones no es otro que tratar de concretar puntos y proyectos comunes que fortalezcan las relaciones eurolatinoamericanas. En este sentido, consideramos que esta tendencia no debe ser vista, de forma simplista, como una debilidad para el avance de la región o un triunfo de los proyectos personalistas de algunos, sino como un fiel reflejo del reposicionamiento de países y regiones ante la gran partida de ajedrez que se juega a nivel mundial. Por lo tanto, consideramos que el gran desafío actual para los países de Alc es no perder de vista el centro de sus prioridades. ${ }^{29}$

Entre los hitos conseguidos en el ámbito de las relaciones bilaterales entre diferentes países de ALC y la UE durante estos años se encuentran los siguientes:

- La entrada en vigor del Acuerdo de Asociación Económica, Concertación Política y Cooperación entre México y la UE.

- El Acuerdo de Asociación Chile-uE.

- El Acuerdo de Asociación Estratégica México-ue y un Plan Ejecutivo Conjunto, México y la UE.

- El Acuerdo de Alianza Estratégica entre la ue y Brasil.

- El Acuerdo de Libre Comercio Multipartes entre la UE-Colombia y Perú.

- La negociación de un Acuerdo de Cooperación Cultural entre la UE-Colombia y Perú.

- Las negociaciones para modernizar el Acuerdo de Asociación Chile-UE, después de 10 años de asociación.

- Las negociaciones para la actualización integral del Acuerdo de Asociación Económica, Concertación Política y Cooperación entre la ue y México, y la extensión del Plan Ejecutivo Conjunto de la Asociación Estratégica.

- Las negociaciones para la profundización de la Alianza Estratégica entre la UE y Brasil.

Así como las relaciones bilaterales de algunos países de ALC con la UE se han consolidado durante este periodo, debemos considerar el lento avance, cuando no fracaso, de las negociaciones de asociación subregional de la uE con Mercosur y

\footnotetext{
${ }^{29}$ CEPAL. La hora de la igualdad. Brechas por cerrar, caminos por abrir, Trigésimo tercer periodo de sesiones de la CEPAL (Brasilia, 30 de mayo- $1^{\circ}$ de junio de 2010), Santiago de Chile, 2010; CEPAL. La UE y alc. Inversiones para el crecimiento, la inclusión social y la sostenibilidad ambiental, Santiago de Chile, 2013.
} 
la can. Los únicos acuerdos conseguidos durante estos años en este ámbito han sido el Acuerdo de Asociación entre Centro América y la uE, el establecimiento del Mecanismo de lnversión del Caribe -creado para apoyar las inversiones europeas en el Caribe- y la conclusión del proceso de ratificación del Acuerdo CAN-UE sobre Diálogo Político y Cooperación.

Esta dificultad para concretar acuerdos a nivel subregional puede responder, entre otros factores, a la actual diversidad de instituciones, foros y proyectos económicos y comerciales subregionales en ALC - el Mercosur, la CAN, la ALBA y la más reciente Alianza del Pacífico-, así como a las diferentes estrategias nacionales de algunos países suramericanos: Venezuela y su recién incorporación a Mercosur; Ecuador y Bolivia - países de la CAN- que negocian también su entrada en Mercosur; Argentina, que trata de proteger sus mercados e industrias frente a terceros, limitando las importaciones de productos de la región; Colombia y Perú, que han firmado acuerdos de libre comercio con diferentes países de la región (por ejemplo, México y Chile), con Eevu y la uE, y Brasil, que juega su propia estrategia para convertirse no sólo en líder regional, sino en consolidar su posición a nivel mundial.

En conclusión, aunque el camino recorrido por la Alianza Estratégica Birregional ha sido positivo en algunos de sus grandes objetivos, se puede seguir mejorando y profundizando en la Asociación. Por ejemplo, cabe preguntarse si en las agendas de las cumbres futuras se podrían implementar, entre otros, a) nuevos mecanismos y estrategias de trabajo birregional; b) nuevas formas de enfrentar los desafíos e intereses comunes y discutirlos, quizá, a un nivel diferente, que no esté supeditado a la celebración de las cumbres, y c) nuevos ámbitos de actuación conjunta que respondan a los desafíos actuales y futuros abiertos por la globalización y las nuevas relaciones de poder entre regiones.

\section{Retos que enfrenta la Alianza Estratégica entre ALC y la UE}

Parece existir un amplio consenso respecto a que las relaciones entre los países de ALC y la uE requieren un nuevo diseño, ${ }^{30}$ con instrumentos, discusión de temas

\footnotetext{
30 Diferentes autores hablan de "un nuevo paradigma" (Gratius, Susanne. Europa y América Latina: la necesidad de un nuevo paradigma, FrIDE, Madrid, 2013); "un nuevo marco de relaciones" (Sanahuja, José Antonio. Hacia un nuevo marco de relaciones entre la UE y ALC, EULAC, Hamburgo, 2013): "una nueva arquitectura" (ARENAL, CELESTINo del. La nueva arquitectura de la integración entre América Latina y el Caribe y las relaciones con la UE, FIIAPP, Madrid, 2010); "bases renovadas" (Bases renovadas para la relación Unión Europea, América Latina y el Caribe, Actas del Seminario UE-LACl GIGA, 17 y 18 de septiembre de 2012, velac, Hamburgo, 2013); "el futuro de las relaciones" (Sanahuja, José Antonio. "El futuro de las relaciones entre la UE y ALC: tres premisas y cuatro proposiciones para el debate", en Bases renovadas para la relación Unión Europea, América Latina y el Caribe, op. cit., pp. 102-111).
} 
y estrategias que sirvan para afrontar los retos actuales y futuros. Veamos brevemente algunos de los factores de cambio.

\section{A) Un contexto internacional cambiante}

Desde el inicio de la crisis financiera en Estados Unidos y su continuación en la UE se ha producido una serie de cambios que deben de tomarse en cuenta: China cada vez más posicionada como potencia desde el punto de vista económico y comercial; el ascenso de los países emergentes (llamados BRIcs y otros incluidos en el G-20) con una gran capacidad y proyección para seguir creciendo y creando riqueza; la UE enfrentada a una de sus peores crisis (económica e institucional) y a una posible pérdida de influencia -económica y política- no sólo en América Latina, sino a nivel mundial, y ALc, que ha experimentado profundos cambios - políticos y económicos- que le han permitido niveles muy interesantes de crecimiento económico durante la primera década del siglo xxl, y que la colocan, junto con los países asiáticos, en una posición muy atractiva de cara a iniciar o ampliar relaciones estratégicas, especialmente con algunos países como México, Brasil, Chile, Perú y Colombia.

\section{B) La nueva dinámica económica y comercial de América Latina y el Caribe}

Las reformas económicas emprendidas desde hace más de una década (caracterizadas por la desregulación de mercados, la liberalización comercial y financiera, la privatización de empresas estatales -especialmente de servicios públicos-, la eliminación de restricciones al capital extranjero, el acceso al capital privado a la explotación de los recursos naturales -especialmente minería-) han permitido que la región sea especialmente atractiva, desde un punto de vista financiero y comercial. ${ }^{31} \mathrm{~A}$ este cuadro de reformas se suma un manejo macroeconómico responsable por la mayor parte de los gobiernos de la región y un contexto económico internacional favorable (un alza de precios de las materias primas que favorece a los países productores de ALC y una persistente crisis financiera en EEUU y la UE, que convierte a ALC en un refugio seguro para los inversionistas). En este contexto favorable, los países de ALC -especialmente algunos como México, Brasil y Colombia- han visto, por ejemplo, cómo los flujos de IED han aumentado de manera sostenida, y las empresas transnacionales han ido adquiriendo cada

\footnotetext{
${ }^{31}$ CEPAL. Inversión extranjera directa y PYMES. Una oportunidad para reforzar los vínculos entre la Unión Europea y América Latina y el Caribe, Santiago de Chile, 2011, p. 12.
} 
vez más importancia en muchas actividades productivas y de servicios. Recordemos además que ALC es una región donde, a pesar de los retos que todavía persisten en cohesión social, se ha avanzado significativamente en la reducción de la pobreza y la ampliación de las clases medias, fenómenos que han permitido también que sus mercados internos san atractivos para las exportaciones de otros países, al aumentar su capacidad de compra y consumo. ${ }^{32}$

\section{c) Las dinámicas internas de integración europea y latinoamericana}

Las dos regiones pasan por momentos diferentes y contrapuestos, ya que, como hemos señalado, al declive económico relativo de los países de la UE -especialmente del sur (Grecia, Portugal, Italia y España) - se contrapone el ascenso económico y comercial de ALc. En esta coyuntura pareciera hasta "normal" modificar algunos de los supuestos que han caracterizado el marco de las relaciones hasta ahora, e introducir, incluso, nuevos temas estratégicos a la Alianza.

En este sentido, se observa un aumento de la visibilidad internacional de ALC, simultáneo a un paulatino declive europeo que exige una nueva mirada a las relaciones birregionales (demasiado focalizadas, hasta ahora, en la cooperación e iniciativas europeas). En este contexto, también debe tenerse en cuenta la existencia de terceros actores interesados en participar del crecimiento y auge económico de ALC, así como aumentar su presencia en algunos países y sectores (véase el caso de China, por ejemplo, sin menospreciar al gran vecino del norte, los EEUU).

Uno de los factores que marcan la dinámica interna de los procesos de integración de ambas regiones es su mayor fragmentación. Esto es evidente en el caso europeo dentro del contexto de crisis financiera y económica que viven sus países ${ }^{33}$ (aunque empieza a preocupar más la crisis institucional y la desafección ciudadana producida por falta de respuestas eficaces de las instituciones comunitarias y la preponderancia de Alemania y sus propuestas de austeridad y recorte)..$^{34}$

En el caso particular de América Latina y el Caribe se ha ido avanzando a una mayor fragmentación regional por motivos diferentes. Desde los años noventa hasta mediados de la década de 2000, los países de ALc conformaron una particular "cartografía de la integración" basada en el "regionalismo abierto", con

\footnotetext{
32 CEPAL. La hora de la igualdad..., op. cit., p. 42.

${ }^{33}$ Véase Pérez Carrillo, Elena F. "Crisis económica y financiera en la ue. Algunas reflexiones en torno a algunos instrumentos estratégicos para la recuperación", en Anuario Mexicano de Derecho Internacional, vol. xl, 2011, pp. 487-511. ${ }^{34}$ Disponible en: http://internacional.elpais.com/internacional/2013/02/15/actualidad/1360957667_144353.html.
} 
cuatro grupos subregionales: ${ }^{35}$ el Mercosur, la can, el Sistema de la Integración Centroamericana -SICA- y la Comunidad del Caribe -CARICOM-, así como dos países -Chile y México-que optaron por seguir su propio rumbo basado en la firma de múltiples acuerdos de libre comercio (modelo que ahora parecen querer reproducir países como Perú y Colombia).

Desde mediados de la primera década de este siglo se han ido consolidado distintas tendencias que, según este autor, ${ }^{36}$ dibujan "nuevas cartografías y estrategias en el regionalismo y la inserción internacional de la región”:

- Observamos, por ejemplo, la paulatina conformación de un área económica norteamericana basada en las preferencias y acuerdos comerciales que vinculan a eEuu, México, Centroamérica y determinados países del Caribe (área en la cual México busca consolidar su papel estratégico tanto a nivel regional e internacional, ${ }^{37} \mathrm{y}$ donde EEuu trata de consolidar su presencia e intereses económicos y comerciales).

- Quizá más interesante, por su novedad y posible alcance, es la apuesta de México, Perú, Colombia y Chile por la Alianza del Arco del Pacífico, ${ }^{38}$ con una marcada orientación hacia el Continente asiático, donde todos sus miembros cuentan con acuerdos de asociación con la uE (hecho que podría significar un potencial en la vinculación entre las dos regiones).

- Por otro lado, hay que destacar la apuesta suramericana representada por un Mercosur ampliado (con la incorporación de Venezuela, y quizá las futuras adhesiones de Ecuador y Bolivia), así como UNASUR y ALBA-TCP. Esta apuesta busca un mayor grado de autonomía regional y mantiene una amplia agenda de políticas regionales de infraestructura y energía.

Como podemos apreciar, en estos momentos la región presenta un panorama bastante fragmentado en cuanto a objetivos y estrategias de integración, lo que se traduce en una dificultad, tanto para determinar estrategias económicas y comerciales conjuntas entre los propios países de ALC, como para avanzar en materia de acuerdos de asociación con otras regiones.

En este sentido, México y Brasil están asumiendo un claro liderazgo en la región y no tienen un rival inmediato en la zona. En términos económicos y

\footnotetext{
35 Sanahuja, José Antonio. op. cit., p. 13.

${ }^{36}$ Ibidem, p. 14.

${ }^{37}$ Disponible en: http://www.eclac.org/cgi-bin/getProd.asp?xml=/prensa/noticias/comunicados/9/49019/P49019. xml\&txsl=/prensa/tpl/p6f.xslEtbase=/tpl/top-bottom.xsl.

${ }^{38}$ Como reflejo de ese interés sólo hace falta mirar el variopinto grupo de países observadores con los que cuenta actualmente la Alianza (Costa Rica, Panamá, Uruguay, Paraguay, Guatemala, España, Portugal, Canadá, Japón, Australia y Nueva Zelanda).
} 
comerciales siguen consolidando su posición: Brasil en el sur del continente y México en América Central y el Caribe. Como dice Susanne Gratius:39

[...] todo ello introduce más incertidumbres que certezas en cuanto al perfil interno y externo de América Latina. El confuso panorama de integración y concertación regional revela, una vez más, un escaso compromiso institucional, la validez del discurso soberanista y las asimetrías existentes entre los países. Si bien los diferentes objetivos y cambios de miembros señalan flexibilidad, restan credibilidad a la integración latinoamericana que siempre ha adolecido de una sólida institucionalidad y de la perspectiva de cumplir con lo anunciado.

En este panorama, no debemos dejar de lado la consideración de algunos retos que enfrentará ALc en los próximos años, y que pueden influir no sólo en la dinámica interna regional sino en sus relaciones con otras regiones (para reforzarlas, en el caso de la UE). Los desafíos a tener en cuenta son, entre otros, la gran dependencia de las exportaciones de materias primas vinculadas a la demanda china y la consecuente desindustrialización; los escasos avances en desarrollo tecnológico y científico, en parte compensados por la creciente demanda de mano de obra profesionalizada, y el desigual crecimiento de los países de ALc. Así, podemos encontrar algunos países de la región con proyecciones bastante atractivas de crecimiento y estrategias claras de internacionalización de sus economías, empresas y mercados -México, Brasil, Chile, Colombia y Perú-, otro grupo con menores tasas de desarrollo, entre ellos Bolivia, otro más inestable en términos políticos y económicos, encabezado por Argentina y Venezuela y, finalmente, los llamados Estados frágiles (Guatemala, Honduras y El Salvador). ${ }^{40}$

\section{Nuevos temas en la agenda de la asociación de la CELAC y la UE}

En este nuevo contexto, la CELAC surge como un nuevo mecanismo que busca ejercer de interlocutor representativo de ALc y avanzar en el objetivo de identificar e impulsar temas de interés regional en los foros globales, a partir de un proceso de concertación y diálogo regional que permita articular las agendas y los trabajos de los distintos espacios subregionales de integración. ${ }^{41}$

\footnotetext{
${ }^{39}$ Gratius, Susanne. op. cit., p. 4.

40 Ibidem, p. 15.

${ }^{41}$ Véase Zabalgoita Trejo, José Antonio. La importancia de la celac y su proyecto de integración con la ue, flacso, San José, 2012, pp. 133-140 (el autor es director general adjunto de Asuntos Hemisféricos y de Seguridad del Gobierno de México).
} 
Este cambio en el diseño de la representación internacional de los países de ALC marca también un giro respecto de las expectativas y valoración de su relación birregional con la UE. Durante años, la agenda entre ambas regiones estuvo marcada por temas de cooperación, con un claro protagonismo de las iniciativas europeas; donde la UE actuó como apoyo y referente político para consolidar en ALc las agendas de paz, institucionalización democrática y desarrollo. En la actual coyuntura, la crisis europea empieza a ser vista como una debilidad de su modelo de integración y como el foco de la recesión económica y de las turbulencias financieras que pueden terminar afectando a los países de ALC.

A pesar de que algunos estudios, como el Informe Prospectivo América Latina, diciembre-enero de $2013 \mathrm{del} \mathrm{Pl}_{1}{ }^{42}$ parecen significar todavía las preferencias de ALC por tener como socio más estratégico a la UE, otros autores ${ }^{43}$ destacan la necesidad de establecer una nueva dinámica que modifique los equilibrios y la tradicional asimetría que durante décadas protagonizó la relación birregional. En este sentido, y como subraya este autor, la uE parece ser una opción menos relevante para la diversificación de las relaciones exteriores de ALC, frente a las oportunidades que se perciben en la región Asia-Pacífico, especialmente de la relación con China,

[...] No se trata tanto de la seducción que pueda emanar del modelo de capitalismo de Estado que está detrás del éxito económico de este país, como del hecho de que en poco tiempo China, un actor menos exigente que la uE en cuanto a su política exterior y su pauta de relacionamiento externo, se ha convertido en el primer socio comercial de algunos países de América Latina y el Caribe, y aunque la UE mantenga

\footnotetext{
42 El Atlas de los intereses entre la Unión Europea y América Latina Caribe, elaborado a lo largo de 2012 por el IPI (Instituto de Prospectiva Internacional) por encargo de la Fundación EULAC, y presentado en la Cumbre CELAC-UE celebrada en Santiago en enero de 2013, señala como datos más significativos los siguientes: "Globalmente, toda América Latina mira a la UE como el socio estratégico más deseado, pero hay diferencias importantes por paises y tipo de actores sociales. Hay seis gobiernos intensamente volcados hacía la UE: Colombia, Costa Rica, Cuba, El Salvador, Honduras y República Dominicana. En un escalón más bajo, pero con bastante interés, están Brasil, Chile, México, Paraguay y Perú. Por debajo, pero todavía con interés por la uE se encuentran Argentina, Bolivia, Haití, Nicaragua y Panamá. Finalmente, hay cuatro gobiernos o presidencias que no están mirando a la UE como socio más deseado: Ecuador, Guatemala, Uruguay y Venezuela. [...] Entre el resto de actores sociales estudiados son las empresas las que se muestran, incluso más que los gobiernos, a favor de la alianza con la uE. No es asi en Colombia, El Salvador, Guatemala y Uruguay. Los medios de comunicación apoyan generalizadamente la alianza con la UE, salvo en Panamá y Paraguay. Finalmente, los ciudadanos también están a favor de manera mayoritaria de que sea la UE el principal referente estratégico de la región, salvo en Bolivia, El Salvador, Guatemala, Panamá y Paraguay. El estudio se realizó analizando más de doscientos setenta y tres millones de búsquedas de los ciudadanos de ambas regiones sobre la otra región, asi como del análisis de contenido de miles de noticias aparecidas en medios escritos sobre la otra región" (ambas investigaciones se basaron en análisis realizados entre octubre de 2011 y octubre de 2012). Disponible en: http://www.minrel.gob.cl/prontus_minrel/site/artic/20130219/asocfile/20130219175745/atlas_de_intereses_entre_la_ue_y_alc.pdf.

43 Sanahuja, José Antonio. Hacia un nuevo marco de relaciones entre la ve y alc, cit., p. 20.
} 
su relevancia como fuente de IED, según distintas proyecciones económicas en pocos años más puede desbancar a la uE del segundo puesto que, tras Estados Unidos, viene ocupando como mercado de destino de las exportaciones de la región.

Este nuevo contexto debería hacer pensar a la uE en cuáles podrían ser las nuevas estrategias y mecanismos que a partir de ahora deben priorizar su relación con ALc, en aras de consolidar la asociación estratégica entre ambas regiones. En cuanto a los países de ALc, es tiempo, también, de aprovechar las ventajas de estas nuevas dinámicas para consolidar planes de crecimiento económico, inclusión social y sostenibilidad ambiental que les permitan cumplir con los enormes desafíos que tienen como región, temas en los cuales su relación con la ue puede ser prioritaria. ${ }^{44}$

Hay pues nuevos temas, nuevos actores, nuevos elementos de agenda y nuevas relaciones de fuerza. En el actual debate relativo a cuáles deben ser los temas y estrategias que deben priorizar la relación birregional encontramos, por ejemplo, visiones absolutamente pragmáticas que abogan por una asociación a diferentes velocidades,

[...] si llegamos a tener acuerdos comerciales ya operativos entre Europa y Chile, Europa y México, y acuerdos a ser aprobados pronto entre Europa, Colombia y Perú así como Europa y Centroamérica, es porque es lo que ha sido posible hacer. Eso es muy importante porque a veces nos imaginamos que tenemos que avanzar todos en la misma dirección, y eso no es posible en la región. [...] Entonces hagamos menús diferenciados de acuerdo a lo que es posible avanzar con cada cual. [...] hemos sabido adaptar la realidad de esa relación a las condiciones específicas en las cuales se puede desarrollar. Hay algunos que pueden ir bastante más lejos en el intento de profundizar nuestros vínculos económicos y comerciales y hay otros que tienen ciertas reservas al respecto. Pues respetemos esa pluralidad y asumamos con pragmatismo un tipo de relación que se ajuste a los requerimientos o necesidades de cada país o grupos de países. Ello resulta inevitable cuando se trata de una región que no tiene una institucionalidad comunitaria alguna como es el caso de América Latina. ${ }^{45}$

Con este mismo sentido pragmático, durante la última cumbre de la CELAC con la UE en la ciudad de Santiago (Chile) se priorizaron, entre otros temas, los negocios comerciales y las prerrogativas de las inversiones. ${ }^{46}$ De esta manera, pareciera que

\footnotetext{
${ }^{44}$ CEPAL. La hora de la igualdad..., op. cit., pp. 149-154.

${ }^{45}$ Garcia Belaunde, José Antonio. Bases renovadas para la relación Unión Europea, América Latina y el Caribe, op. cit., pp. 82 y 83.

${ }^{46}$ Declaración Empresarial. Iv Cumbre Empresarial UE-ALc. Inversiones para el crecimiento económico, la inclusión social y la sustentabilidad ambiental. Santiago de Chile, 26 de enero de 2013.
} 
la iniciativa privada y empresarial empujara las decisiones de algunos gobiernos hacia una mayor coordinación y apertura para profundizar en los lazos económicos y comerciales entre ambas regiones (recordemos que la UE invirtió durante la última década 30 mil millones de dólares anuales en ALc, lo que representa cerca del 40\% del total de sus inversiones en el extranjero, según el lnforme de Inversiones Extranjeras en ALc de la CEPAL en 2011). En este sentido, no es extraño que en la agenda de debate de la última cumbre se incluyera un punto acerca del papel de las inversiones asociadas a la responsabilidad social empresarial y al emprendimiento como motor de desarrollo, mostrando de esa manera el interés por seguir atrayendo más inversión de calidad hacia ALc. ${ }^{47}$

Así pues, los puntos de mayor interés en la actual agenda económica birregional son "la inversión, la seguridad jurídica asociada a ella (tema prioritario para la UE), el comercio, el desarrollo sostenible y la responsabilidad social de las empresas". Estos temas, junto a otros - ciencia, tecnología, innovación, energía y medio ambiente-, forman parte de un paquete de nuevos temas estratégicos que seguro van a protagonizar las próximas reuniones birregionales ${ }^{48}$ (temas llamados a relanzar e impulsar el marco de asociación entre los países de ALC y la UE en los próximos años). Hablamos, pues, de una "amplia agenda de cooperación temática avanzada", ${ }^{49}$ de "un menú a la carta sin tabúes" ${ }^{50}$ acorde a las nuevas demandas que presentan ambas regiones.

Uno de los temas estratégicos de mayor interés mostrado en las últimas reuniones de gobiernos y sectores privados de ALC-UE es la "cooperación empresarial". Recordemos que las pequeñas y medianas empresas (PYMEs) representan un porcentaje muy importante de la actividad empresarial en la UE y ALC, y generan una importante tasa del empleo en sus países (un 67\% según los datos mostrados en la Cumbre Empresarial de Santiago) y se desarrollan en una gran diversidad de ámbitos de la producción y de los servicios. Las principales diferencias entre las PYMES de ambas regiones son aquéllas relativas a los niveles de internacionalización y de encadenamientos productivos, niveles de productividad y su aporte al PIB.

Dado que la uE es, por ahora, la principal fuente de inversión extranjera directa para ALc, ésta debería convertirse en una oportunidad para seguir mejorando la competitividad de las PYMEs mediante la cooperación empresarial, es decir, pro-

\footnotetext{
47 CEPAL. La hora de la igualdad..., op. cit., pp. 13-15.

48 De gran interés serán las relaciones en las áreas de las energias renovables, tecnologias limpias, economía verde y biotecnología; aprovechando las ventajas competitivas y tecnológicas que pueden surgir de la cooperación entre empresas de ambas regiones.

${ }^{49}$ Véase Sanahuja, José Antonio. Hacia un nuevo marco de relaciones entre la Ue y alc, cit., pp. 27 y 28.

50 Véase Gratius, Susanne. op. cit., pp. 18-20.
} 
moviendo nuevos encadenamientos productivos entre empresas multinacionales y otras de menor tamaño. En este sentido, uno de los grandes retos de las PYMES de ALc en los próximos años es mejorar sus niveles de productividad, incorporando innovación tecnológica en áreas con proyección de crecimiento, tarea en la cual puede interesar que se cuente con la cooperación de la UE, ya que esas áreas son aquéllas de mayor interés para las IED en países que ofrecen seguridad jurídica y garantías para desarrollos de mediano y largo plazo.

Junto a esta interactuación entre "productividad empresarial-innovación tecnológica-IED” (donde el papel de la cooperación empresarial entre la UE y ALC puede ser muy interesante), las PYMEs necesitan, además, políticas públicas nacionales específicas y adaptadas a la capacidad de las PYMEs, dirigidas a fomentar el emprendimiento y la internacionalización, y que incentiven la actividad empresarial privada.

Algunos de los principales problemas que presentan las PYMES -especialmente en ALC- son su escasa competitividad, poca innovación, baja productividad y empleos mal remunerados. En este sentido, desde los propios sectores empresariales implicados, se recomiendan varias acciones de cooperación entre ambas regiones, entre ellas:

- Buscar amplios consensos entre las instituciones públicas y privadas para avanzar en cuatro áreas interrelacionadas de políticas de apoyo: a) innovación para el fortalecimiento de capacidades productivas y gerenciales; b) acceso a mercados; c) articulación productiva y cooperación empresarial, y d) acceso al financiamiento.

- La implementación de acciones en tres grandes áreas de políticas: a) la capacitación y sensibilización de las PYMEs para la internacionalización; b) la promoción de encadenamientos productivos y la identificación de oportunidades de negocios con empresas transnacionales y cadenas globales de valor, y c) la facilitación del encuentro y la búsqueda de cooperación directa entre PYMES de la UE y ALc.

- Estas acciones deberían reforzarse con la entrega de información y la realización de búsqueda de oportunidades de mercados y posibilidades de colaboración y asociación, y la promoción de esquemas de complementariedad entre empresas, así como el mejoramiento de la visibilidad y difusión de los programas de apoyo.

Debemos resaltar, finalmente, la importancia de seguir trabajando en el desarrollo de instrumentos y programas que crean sinergias y favorecen e impulsan esta colaboración empresarial eurolatinoamericana a través de acciones de apoyo a las 
PYMES de ambas regiones y la creación de redes de organizaciones intermediarias - por ejemplo, el programa AL-Invest, la red Enterprise Europe Network, el Export Help Desk o los Programas Marco de Investigación financiados por la UE-.$^{51}$

\section{Conclusiones}

1) Cumbres birregionales. La celebración de las cumbres birregionales ha servido para estrechar las relaciones entre los países de ALC y la UE sobre la base de valores compartidos (la democracia y la defensa del multilateralismo en las relaciones internacionales, el Estado de derecho y la seguridad jurídica), así como para construir una asociación estratégica que privilegia la cooperación entre ambas regiones. La Alianza ALC-UE, a pesar de todos los inconvenientes que pudieran haberse cruzado en el camino estos últimos años, ha permitido articular una serie de mecanismos y ejecutar una serie de acciones de cooperación política, económica y comercial que han resultado positivos entre ambas regiones, en un contexto mundial cambiante y bastante complejo, caracterizado por una recomposición de poderes donde la conformación de alianzas estratégicas entre regiones resultará fundamental.

2) Asociación Birregional. Como hemos podido ver a lo largo del artículo, la Asociación ALC-UE es útil y buena para los propios intereses de los países de ALc, ya que les está permitiendo proyectar el papel de la región y sus prioridades (en ocasiones también sus diferencias) ante terceros, consolidar sus estrategias de negociación ante otras regiones, armonizar entre sí sus posiciones a través del diálogo y hacerlas compatibles con sus propios proyectos subregionales, sin que por ello se deje de avanzar en temas de la agenda bilateral y tomar decisiones concretas al respecto.

3) Alianza Estratégica. Este instrumento ha permitido conseguir una serie de avances positivos que han reforzado el diálogo y la coordinación ALC-UE a través de mecanismos y lineas de acción en diferentes políticas, como por ejemplo en el ámbito educativo: el Espacio ALC-UE del conocimiento en materia de enseñanza superior, el programa $A L \beta_{A N}$ de enseñanza superior y la Iniciativa Conjunta de Investigación e Innovación; en el ámbito social: el programa EUROSOciAL; en el ámbito medioambiental: el programa EUROCLIMA; en el ámbito de la comunicación y las redes digitales: el programa @lis;

\footnotetext{
${ }^{51}$ De gran interés por la estrecha colaboración que se puede desarrollar entre instituciones académicas, sector privado y entes gubernamentales de ambas regiones.
} 
en el ámbito financiero y económico: los Mecanismos de lnversión para América Latina y el Caribe, y en el ámbito de la cooperación parlamentaria: la Asamblea Parlamentaria Euro-Latinoamericana (EuroLat).

4) Empuje comercial. Otro de los ámbitos donde mayor nivel de diálogo y negociación ha habido entre la UE y algunos países de ALc ha sido el comercial, a través de la firma de acuerdos de asociación y libre comercio, especialmente a nivel bilateral: el Acuerdo de Asociación Económica, Concertación Política y Cooperación entre México y la UE; el Acuerdo de Asociación Chile-ue; el Acuerdo de Asociación Estratégica México-ve y un Plan Ejecutivo Conjunto, México y la UE; el Acuerdo de Alianza Estratégica entre la UE y Brasil; el Acuerdo de Libre Comercio Multipartes entre la UE-Colombia y Perú, y el Acuerdo de Asociación entre Centro América y la UE.

5) Planes de acción. La Alianza Estratégica Birregional se ha visto reforzada por la implementación de dos planes de acción para tratar de consolidar líneas de actuación concretas y ampliar los ámbitos de trabajo conjunto ALC-UE: el Plan de Acción ALC-UE 2010-2012, con medidas concretas financiadas y definidas mediante una agenda concreta y práctica en ámbitos prioritarios como el desarrollo del espacio UE-ALC del conocimiento, el fomento de la interconectividad y la intensificación de los diálogos temáticos y la cooperación sobre migraciones, drogas, educación y cohesión social, y el Plan de Acción CELAC-UE 2013-2015, que a los temas anteriores suma dos: "Género e inversiones y emprendimiento para el desarrollo sustentable".

6) Diálogo político ALC-UE. Es otro de los ámbitos que ha tenido un interesante desarrollo durante este periodo, abriendo camino a una serie de foros eurolatinoamericanos para el diálogo y la cooperación en diferentes ámbitos y niveles (las reuniones de altos funcionarios de gobierno, foros parlamentarios, sindicales, académicos, empresariales, judiciales y de la sociedad civil). Cada uno de ellos, en su ámbito, ha permitido que representantes de ALC-UE expresen sus ideas, intereses, retos y propuestas de una manera fluida y coordinada, en busca de un mejor entendimiento de las relaciones y de propuestas que profundicen los vínculos bilaterales entre ambas regiones.

7) Bilateralidad. En la actualidad se percibe un creciente uso de la bilateralidad entre algunos países de ALc y la uE, a través de las asociaciones estratégicas y/o los acuerdos de libre comercio como los que la ue ha firmado con México, Brasil, Chile, Colombia y Perú. Tendencia que también se refleja a través de las asociaciones especiales entre algunos Estados miembros de la UE -España, Italia, Francia, Alemania- con determinados socios latinoamericanos. Creemos, pues, que el bilateralismo dominará frente a los 
esquemas interregionales que se mantendrán para los socios más pequeños (CARICOM Y SICA).

8) Factores de cambio. Consideramos que el nuevo diseño del Marco de Asociación ALC-UE debe afrontar retos actuales y futuros. Éstos se observan mediante el análisis de un contexto internacional cambiante, la nueva dinámica económica y comercial de América Latina y el Caribe, y las dinámicas internas de integración europea y latinoamericana.

9) Mayor equilibrio y simetría. Es necesario establecer una nueva dinámica que modifique los equilibrios y la tradicional asimetría que durante décadas protagonizó la relación birregional. En este sentido, la UE deberá tener en cuenta el cada vez mayor interés de los países de ALc en diversificar sus relaciones exteriores (especialmente con los países de la región Asia-Pacífico). Es por ello que los países europeos deberán implementar mecanismos que sigan haciendo atractiva a la UE como socio estratégico frente a otros posibles competidores.

10) Desafíos pendientes. Creemos primordial para relanzar las relaciones ALCUE: coordinar de una manera más eficaz y resolutiva las agendas, y que el diálogo entre regiones sea más fluido y constante, basado en el equilibrio entre las partes, para así: $a$ ) encontrar nuevos mecanismos y estrategias de trabajo birregional que permitan enfrentar los desafíos e intereses comunes y discutirlos, quizá a un nivel diferente, que no esté supeditado a la celebración de las cumbres, y b) implementar nuevos ámbitos de actuación conjunta que respondan a los desafíos de la globalización y las nuevas relaciones de poder entre regiones.

11) Nuevos temas en la agenda. Finalmente, hemos visto que las iniciativas público-privadas adquieren un papel cada vez más activo en el devenir y el fortalecimiento de las relaciones ALC-UE. Así, hemos subrayado la importancia de la incorporación de temas que afianzan la agenda económica birregional y sirven de impulsores de nuevos programas y líneas de cooperación entre ambas regiones; por ejemplo, la inversión y la seguridad jurídica asociada a ella; los flujos comerciales; el desarrollo sostenible; la cooperación empresarial y la responsabilidad social de las empresas; la cooperación científica y tecnológica; la innovación; la energía y el medio ambiente. Todos estos temas son estratégicos no sólo para el desarrollo económico y social de cada país, sino para el posicionamiento en el mundo; es por ello que deben protagonizar las agendas y acuerdos de las próximas reuniones birregionales. Consideramos que son los temas que pueden relanzar e impulsar el marco de asociación entre los países de ALC y la UE en los próximos años. 